\title{
Germanica
}

\section{Die didaktische Pragmatik und ihre Grenzen im Zeitroman von Siegfried Lenz}

La didactique pragmatique et ses limites dans les romans d'actualité de Siegfried Lenz

\section{Elfie Poulain}

\section{OpenEdition}

\section{Journals}

Édition électronique

URL : http://journals.openedition.org/germanica/2219

DOI : 10.4000/germanica.2219

ISSN : 2107-0784

\section{Éditeur}

Université de Lille

\section{Édition imprimée}

Date de publication : 1 décembre 1994

Pagination : 123-135

ISSN : 0984-2632

Référence électronique

Elfie Poulain, « Die didaktische Pragmatik und ihre Grenzen im Zeitroman von Siegfried Lenz »,

Germanica [Online], 14 | 1994, Online erschienen am: 20 Januar 2014, abgerufen am 06 Oktober 2020.

URL : http://journals.openedition.org/germanica/2219; DOI : https://doi.org/10.4000/germanica.2219

Ce document a été généré automatiquement le 6 octobre 2020.

(c) Tous droits réservés 


\section{Die didaktische Pragmatik und ihre Grenzen im Zeitroman von Siegfried Lenz}

La didactique pragmatique et ses limites dans les romans d'actualité de Siegfried Lenz

Elfie Poulain

1 In seinem Buch Beziehungen. Ansichten und Bekenntnisse zur Literatur betont Siegfried Lenz, daß Geschichte keineswegs der objektive Stoff sei, der in den Verliesen der Zeit ruhe. "Von der Geschichte», so schreibt er, "geht vielmehr eine permanente Herausforderung aus, die jeden in seiner Gegenwart betrifft, die jeden zwingt, seine Fragen an vergangene Begebenheiten $\mathrm{zu}$ stellen. Insofern ist Geschichte eine Möglichkeit zum Selbstverständnis» ${ }^{1}$. Dieses Zitat macht zweierlei deutlich: zum einen die enge Verbindung, die Lenz zwischen Literatur und Geschichte aufstellt, d.h. für ihn vornehmlich Geschichte seiner Zeit, zum anderen zeigt es seine Auffassung von zeitgeschichtlichem Geschehen als Offenbarungsmöglichkeit von Bewußtseinsstrukturen. Unter diesem Aspekt ist für S. Lenz Geschichte identisch mit dem, was Alexandre Kojève in seiner Hegel-Interpretation über Geschichte lehrt: Geschichte ist keine Aneinanderreihung von empirischem Material, von historischem Stoff; Geschichte ist immer menschliche Geschichte. Natur hat keine Geschichte. Geschichte ist die Geschichte menschlichen Handelns, menschlichen Werdens. Sie zeugt vom Wandel des menschlichen Geistes, von seiner Selbstverwirklichung in Raum und Zeit².

2 Mit Hilfe seines Schreibens versucht S. Lenz, diesen Wandel des menschlichen Bewußtseins $\mathrm{zu}$ veranschaulichen und eine Verbindung aufzustellen zwischen Wirklichkeit und Imagination. Denn die Vorstellungen in unserem Kopf, unsere Wünsche und Ideale bedingen eben jene Ereignisse, die wir Geschichte oder Zeitgeschehen nennen. "Als Geschichtenerzähler kommt man nicht darum herum, nach Motiven, Gründen zu fragen, die uns so oder so handeln lassen», bekennt S. Lenz ${ }^{3}$. Seine Romane Deutschstunde $e^{4}$ und Heimatmuseum ${ }^{5}$, auf die sich die gegenwärtige Analyse 
stützt, spielen im Deutschland der Nazizeit und der Nachkriegsjahre, d.h. in einer Zeit, die der Autor selbst als junger Mensch miterlebt und miterlitten hat. Das Anliegen des Autors besteht darin, auf dem Hintergrund jener zeitgeschichtlichen Ereignisse und des daran anknüpfenden fiktiven, literarischen Geschehens die Motivationen, d.h. die geistigen Strukturen zu ergründen, die in den Entscheidungsprozessen wirksam sind und die Handlungen seiner Romangestalten, wie die der Menschen insgesamt, orientieren.

Im folgenden möchte ich aufzeigen, inwiefern bei S. Lenz diese EntScheidungsprozesse im Roman Deutschstunde auf der Identifizierung mit der sozialen Rolle beruhen und wie diese Identifizierung ein Verhalten auslöst, das zu jenen fatalen geschichtlichen Konsequenzen geführt hat, deren Vergangenheit noch nicht vergangen ist. Der folgende Roman, Heimatmuseum, bildet insofern eine Fortsetzung der Problematik als er aufzeigt, wie der Romanheld durch kritisches Denken zu einer Distanzierung vom Ideal der Rolle gelangt, dadurch aber in einen neuen Bewußtseinskonflikt gerät. Schließlich möchte ich die Frage aufwerfen, welche Wirkung die den Romanen innewohnende Didaktik auf den Leser auszuüben vermag, dem die literarische Zeitkritik des Autors nicht entgehen kann.

Der 1968 veröffentlichte Roman Deutschstunde spielt in einem kleinen Landbezirk Norddeutschlands in den Jahren 1943 bis 1954. Er veranschaulicht die verhängnisvollen Folgen, die die blinde Identifizierung des Jens Ole Jepsen mit seiner Rolle als Polizist für seine Mitmenschen und für sich selbst nach sich zieht. Ausgangspunkt der Handlung und des Konflikts bildet das von den Nazis verhängte Malverbot, das der Polizist Jepsen im Jahre 1943 seinem Jugendfreund, dem expressionistischen Maler Max Ludwig Nansen überbringen und das er auch überwachen muß. Dieser Konflikt weist auf das über Emil Nolde verhängte Malverbot, und ganz allgemein auf die von den Nazis besonders verfolgten Expressionisten zurück. Beide Romangestalten, der Polizist und der Maler, bilden ein Gegensatzpaar. Jepsen ist Repräsentant einer totalitären Macht, d.h. der Naziherrschaft, der er als Staatsbeamter dienen muß, und Nansen ist Repräsentant der Kunst, d.h. eines freischaffenden, liberalen Geistes. Wichtig ist hier die Tatsache, daß sich beide ausnahmslos mit ihrer sozialen Rolle identifizieren. Sowohl der Polizist als auch der Maler geben sich von vornherein schon in ihrer äußeren Erscheinung als Polizist bzw. als Maler zu erkennen. Jepsen erscheint immer und überall in seiner straff sitzenden, überaus korrekten Uniform, Nansen seinerseits zu jeder Jahreszeit, draußen und drinnen, in seinem abgetragenen, weiten Mantel mit dem saloppen Filzhut (cf. DEU 24-25, 332). Rein äußerlich also tragen sie ihre innere Anschauung von sich selbst zur Schau. Die enge Uniform scheint Jepsens Engstirnigkeit zu entsprechen und der weite Künstlermantel für Nansens Forderung zu stehen, man müsse weiter sehen, über den begrenzten Horizont des eigenen Bezirkes hinausschauen. Der Romanverlauf zeigt, wie ihre Einstellung zu sich selbst ihr Verhalten im sozialen System bedingt, insofern es ein Pflichtdenken veranschaulicht, $\mathrm{da}$ an die Rechte und Pflichten bzw. die Erwartungen anknüpft, die mit ihrer sozialen Rolle verbunden sind. Das Pflichtdenken von Jepsen beruht auf dem Bild, das er sich vom idealen Polizisten macht. Anfänglich ruft das Malverbot, das er seinem Jugendfreund überbringen soll, zwar Befremden in ihm hervor, doch unterdrückt er seine Zweifel allsbald, indem er sich seine Pflicht als Polizist vor Augen ruft. Jepsen ist in einem autoritätsgläubigen Milieu aufgewachsen. Er hat das traditionelle Denken und die kleinbürgerlichen Verhaltensnormen vorbehaltlos übernommen und nie erwogen, die Macht je in Frage zu stellen. So gehorcht er auch in diesem Fall aus Gewohnheit und 
anerzogenem Gehorsam, obwohl er nichts von Kunst versteht, folglich nichts von dem, was die Expressionisten als ihre Aufgabe betrachten und was sie zu Verfolgten des Naziregimes macht. Wie manch einer seiner Generation, illustriert er ein Verhalten, das Karl Marx folgendermaßen beschreibt: «Die Bürokratie ist eine Hierarchie des Wissens. Die Spitze vertraut den unteren Kreisen die Einsicht ins Einzelne zu, wogegen die unteren Kreise der Spitze die Einsicht in das Allgemeine zutrauen, und so täuschen sie sich wechselseitig» ${ }^{6}$. Als Untergebener eines totalitären Regimes kann Jepsen nicht umhin, den Befehl als solchen auszuführen, doch bleibt es ihm als einzigem Repräsentanten dieses abgelegenen Amtsbezirkes überlassen, die Art und Weise zu bestimmen, wie er den Maler zu überwachen hat. Bedeutsam ist, wie er den ihm überlassenen Freiraum interpretiert. Gerade hier aber zeigt er, in welchem Maße er seine Rolle als Norm und Komplex von Erwartungen betrachtet, was ihm die Sicht für die gegebene Situation verstellt. Wenn immer Gegenstimmen im Dorf laut werden und sagen, er tue mehr als seine Pflicht (cf. DEU 93-94), wenn immer der Maler versucht, an seinen Verstand zu appellieren («Mein Gott, Jens, wann wirst du merken, daß sie Angst haben und daß es die Angst ist, die ihnen rät, sowas zu tun: Berufsverbote auszusprechen und Bilder zu beschlagnahmen», DEU 67), dann entgegnet er nur stur und gelassen: «Is in Berlin verfügt worden, das genügt». (Deu 67). Für ihn gilt die den Deutschen so fatal gewordene Maxime: Befehl ist Befehl. Was er von sich selbst fordert, das fordert er auch von allen anderen. In diesem Sinne befiehlt er seinem Sohn: «Du brauchst nicht mehr zu verstehen als du gesagt bekommst, das genügt... Brauchbare Menschen müssen sich fügen» (DEU 53).

5 Ein solches Pflichtdenken ist jedoch demjenigen des Malers polar entgegengesetzt. Dieser sieht seine Rolle als Künstler darin, die Wahrheit über sich und seine Umwelt darzustellen. Das bedeutet im vorliegenden Fall, die Furcht und den Schrecken im Bilde festzuhalten, die er, der Maler, in den Gesichtern seiner Mitmenschen sieht. Er kann also, so lange er am Ideal seines Künstlertums festhält, weder aufhören zu malen noch das malen, was Adolf Ziegler vom Haus der Deutschen Kunst zu sehen wünscht (cf. DEU 301). Seine zeitkritische Einstellung als Künstler wird nach dem Krieg hervorgehoben, wenn er erklärt, er «habe diesen politischen Spuk auf seine Weise dargestellt: ihm sei es darauf angekommen, auf die Beziehung zwischen Außenwelt und Bildwelt hinzuweisen» (DEU 302).

Die Rollenidentifizierung und das damit verbundene Pflichtdenken sind entscheidend für die Dynamik des Romangeschehens: Jepsens Verhalten ist bestimmt durch sein Missionsbewußtsein als getreuer Befehls Vollstrecker, Nansens Verhalten hingegen durch sein Bewußtsein als Aufklärer des Volkes. Die Erwartungen, die jeder der beiden Romangestalten in den anderen setzt, erweisen sich als Projektionen des Bewußtseins, das ihr eigenes Verhalten motiviert. So erwartet der Polizist, daß Nansen das Malverbot anerkennt und nicht mehr malt. Der Maler seinerseits erwartet, daß Jepsen ihn nicht so streng überwacht und daß er im geheimen weitermalen kann. Und so werden beide in ihren Erwartungen getäuscht. Aus der rollengemäßen Reaktion des einen ergibt sich die erbitterte Gegenreaktion des anderen. Nansen erklärt auf das Malverbot und die Beschlagnahmung seiner Bilder hin, er werde weitermalen, unsichtbare Bilder malen. Das aber faßt der Polizist als persönliche Beleidigung und Provokation auf. Er konfisziert Nansens Mappe mit den unsichtbaren Bildern (die in der Tat nur weiße Blätter enthält), bespitzelt ihn mehr denn je, bei Tag und bei Nacht, und erstattet schließlich Anzeige, was zur Verhaftung des Malers führt. So entwickelt sich der Konflikt crescendo. Die anfängliche Identifizierung steigert sich zur Idealisierung der 
jeweiligen Rolle und hat die gnadenlose Verfolgung seitens des Polizisten und den unerbittlichen Widerstand seitens des Malers zur Folge. Individuelles Bewußtsein und soziales Handeln werden hier in ihrer Wechselwirkung deutlich mit all ihren verheerenden Folgen im zeitgeschichtlichen Kontext.

Der Autor zeigt in diesem Roman, wie Pflichtbewußtsein in Pflichtfanatismus ausarten kann, wie sich der anfänglich gutmütige und gewissenhafte Polizist infolge der Idealisierung seiner Rolle und der ihr innewohnenden grenzenlosen Autoritätsgläubigkeit zu einem kritiklosen Untertan, zu einem blinden Werkzeug der Machthaber und d.h. zu einem Mitläufer entwickelt, der schließlich seinem eigenen Verfolgungswahn zum Opfer fällt. In der Person von Jepsen entdecken wir jenen gehorsamen Menschentyp, den die deutsche Geistesgeschichte durch Jahrhunderte hindurch geprägt hat. Jepsen vertritt starrsinnig eine allgemeine Ordnung, ohne deren Legitimität, Ziele und Vernünftigkeit in Frage zu stellen. Im Gegensatz zu Nansen, der wiederholt an das eigene Gewissen, die eigene Verantwortung appelliert und betont, man müsse voraussehen, «voraussehen, wozu eine Sache führt» (DEU 156), beruft sich der Polizist in seiner Verblendung stets auf Vorschriften, die ihm von außen bzw. von oben diktiert werden anstelle seine eigene Vernunft kritisch zu befragen. Das aber entspricht keineswegs dem allgemeinen Gesetz, auf das Kants kategorischer Imperativ aufbaut, sondern vielmehr einer Perversion desselben. Deshalb muß Jepsen als unmündiger, unvernünftiger Mensch betrachtet werden, der umso gefährlicher ist als er in seiner Rolle als Polizist mit Macht und Autorität über die Mitmenschen ausgestattet ist. Die Pragmatik des Romans läßt die Heranbildung jener Bewußtseinsstrukturen erkennen, die über die individuellen Handlungen hinaus auch die geschichtlichen Handlungen mitbedingen und $\mathrm{zu}$ jenen fatalen Gesinnungen führen, die den Faschismus möglich gemacht haben.

8 Anders verhält es sich im Roman Heimatmuseum, der im Jahre 1978 veröffentlicht wurde. In der Gestalt des Erzählers Zygmunt Rogalla zeigt der Autor einen geschichtsund ideologiebewußten Menschen, der aus der Geschichte seiner Väter gelernt hat und den Gefahren einer blinden Rollenidentidentifizierung zu entgehen versucht. Der Roman spielt in der Gegenwart im nördlichen Deutschland und stellt eine Rückblende auf mehr als sechzig Jahre deutscher Zeitgeschichte dar. Der Erzähler berichtet von seinem Heimatland Masuren im südlichen Ostpreußen, von der Vertreibung während des Zweiten Weltkrieges und der Wiedererrichtung seines privaten Heimatmuseums im Westen, das die mühsam geretteten Schätze aus dem Osten nunmehr wieder öffentlich ausstellt. Zeitgeschichtlich gesehen fällt das Schreiben dieses Romans in die Epoche der Aussöhnungspolitik mit dem Osten. Der aus Ostpreußen stammende Autor war damals (1970) mit Bundeskanzler Willy Brandt und Günter Grass zur Unterzeichnung des deutsch-polnischen Vertrages über die Anerkennung der Oder-Neiße-Linie nach Warschau gereist.

9 Wie in Deutschstunde, so ist auch Zygmunt Rogalla in Heimatmuseum eine Person, die ihren Beruf als Treuhänder der Museumsschätze durchaus als Berufung im Sinne protestantischer Ethik auffaßt, d.h. als Lebensrolle und Lebensaufgabe. Wie sehr sich Zygmunt mit seiner sozialen Rolle identifiziert, zeigt allein die Tatsache, daß er durch all die Wirrnisse von Krieg und Vertreibung hindurch das für ihn Wertvollste rettet, nämlich einige Kisten mit den Museumsschätzen, die er in seinem neu errichteten Museum im Westen ausstellt. Die Achtung des überkommenen Erbgutes und die innere Wahrhaftigkeit den Zeugen der Vergangenheit gegenüber ist dem Erzähler oberstes 
Prinzip. Diese Auffassung bringt ihn jedoch sowohl unter den Nazis in Masuren als auch nach dem Krieg in Norddeutschland in Bedrängnis, insofern das überkommene Erbgut teils deutschen und teils polnischen Ursprungs ist. Ähnlich wie in Deutschstunde, so bewirkt auch in diesem Roman die Interaktion mit seinem Freund und Gegenspieler Conny Karrasch die Zuspitzung des Konfliktes, der wie zuvor durch den zeitgeschichtlichen Kontext und die Idealisierung der sozialen Rolle ausgelöst wird. Zwei Episoden machen deutlich, daß der Erzähler hier Pflichtbewußtsein und Notwendigkeit der Distanzierung zu vereinen vermag. Während des Krieges in Masuren legt Zygmunt eine heimattreue Gesinnung an den Tag, die der propolnisch auftretende Conny Karrasch nur mißbilligen kann. So weist er Zygmunt mit der Bemerkung zurecht, er «mache aus dem Heimatgedanken eine Religion» (HEIM 232). «Du hast vergessen», sagt Conny, «du hast den Haß vergessen, Zygmunt, der aus all dem kommt, was du Heimat nennst, den Haß auf die anderen» (HEIM 233). Wenig später wird Zygmunt mit einem Befehl konfrontiert, der seine innere Wahrhaftigkeit auf die Probe stellt. Die Nazioffiziere verlangen von ihm, aus seinem Heimatmuseum in Masuren ein Grenzmuseum zu machen und alle Funde polnischen Ursprungs auszusondern. Im Gegensatz zum regimetreuen Polizisten Jepsen in Deutschstunde beugt sich Zygmunt jedoch nicht dem Befehl, weil dieser seinem Gerechtigkeitssinn widerspricht. Deshalb beschließt er kurzerhand, sein Museum zu schließen. Innere Aufrichtigkeit seinem Ideal als Treuhänder der Vergangenheit gegenüber bestimmen seine Handlung. Sie ist ihm wichtiger als ein äußeres, verfälschtes Fortbestehen seiner historischen Zeugen und seiner damit verbundenen sozialen Rolle.

10 Nach dem Krieg erfährt Zygmunt Ähnliches im Westen. Diesmal jedoch, und allen Erfahrungen zum Trotz, sind es Conny Karrasch und der Heimatverein, die Grund zum großen Sortieren sehen. Sie wünschen «eine fleckenlose Geschichte ... die keinen belastete» (HEIM 645) und bedrängen Zygmunt, die Propagandaplakate für Polen und ähnliche Anlässe zu Mißdeutungen aus dem Museum zu entfernen, andernfalls würde man die Zuwendungen streichen. Wie ehedem, so weigert sich auch Zygmunt hier, dem ihm irrig erscheinenden Wunsch nachzukommen. Stets war er darauf bedacht gewesen, die Zeugen der Vergangenheit selbst und uneingeschränkt sprechen zu lassen. Eine derartige Aussonderung würde in seinen Augen bedeuten, die Vergangenheit zu fälschen und Mißbrauch mit ihren Zeugen zu treiben. Rätselnd über «die nur befristete Dauer unserer Grundsätze» (HEIM 650) sieht Zygmunt sich zu einer Radikallösung getrieben: sein Museum endgültig zu zerstören, in Brand zu setzen, und folglich seiner sozialen Rolle und d.h. seiner Lebensaufgabe, ein Ende zu setzen. Sein Handeln ist für ihn selbst ein heroisches Handeln, das zwar das äußere problematische Objekt vernichtet, nicht aber den Konflikt als solchen. Denn sein Problem bleibt in Form eines psychologischen Rollenkonflikts bestehen, nämlich als Bewußtseinskrise, insofern sein privates Handeln zugleich auch ein öffentliches Handeln ist. Es betrifft alle Vertriebenen, all jene, denen er durch sein Handeln die Zeugen ihrer nationalen Identität raubt.

11 Auch dieser Roman macht deutlich, wie sehr zeitgeschichtliches Handeln durch die Bewußtseinsstrukturen der Handlungsträger bedingt ist. Anders als im Roman Deutschstunde, in dem die Romangestalten ungeachtet der zeitgeschichtlichen Machtverhältnisse ein starres Festhalten am Ideal der eigenen Rolle illustrieren, erweist sich Zygmunt in Heimatmuseum als fähig, sich im entscheidenden Moment vom Ideal seiner Rolle loszusagen, die zweifellos seinen Lebensinhalt darstellt. Trotz seines stark ausgeprägten Heimatsinnes bleibt er der geschichtlichen Entwickhing gegenüber 
klarsichtig. So sehr er auch die Mentalität seiner Mitmenschen versteht, die ihre Vergangenheit im Osten zu verklären suchen, so sehr ist er sich dennoch bewußt, daß ein Aussortieren der Museumsobjekte ein Verrat an der Vergangenheit wäre. Vergangenheit, sagt er, «gehört uns allen, man kann sie nicht aufteilen, zurechtschleifen» (HEIM 419). Sein radikaler Entschluß, alles zu zerstören, wurzelt in einem ideologischen Motiv: Zygmunt wehrt sich gegen jegliche Art von Manipulation, die eine authentische Erinnerung an die Vergangenheit, und somit die Wahrheit der Geschichte, zu verfälschen trachtet. Er mag nicht dulden, daß man sein Museum in den Dienst einer Idee stellt und zu Propagandazwecken mißbraucht, sei es in Masuren während des Krieges oder in Norddeutschland nach dem Krieg. Es würde bedeuten, daß man den geschichtlich überlieferten Dingen den ideellen Wert raube und sie zu Konsumartikeln erniedrige; daß man sie dazu ausnutze, das Bewußtsein der Öffentlichkeit zu beeinflussen, was im gegebenen Fall die Frage der Teilung der deutschen Ostgebiete anbetrifft. Darüber hinaus zeigt die Problematik Zygmunts, daß nicht die Museumsobjekte allein ihrem Zweck entfremdet würden. Auch er selbst, als Person, würde hierdurch zu einem dienstbaren Objekt degradiert und folglich sich selbst und seinem wahren Auftrag entfremdet werden. Zygmunt erkennt, was Jepsen in Deutschstunde nicht zu erkennen vermochte: daß man den tatsächlichen Bezug zur objektiv erlebten Wirklichkeit anerkennen und das idealisierte Bild der eigenen Rolle in Frage stellen muß, wenn es die Wahrheit sich selbst und dem Zeitgeschehen gegenüber erfordert. In diesem Sinne führt er aus, was schon der Maler in Deutschstunde erbittert hervorgestoßen hatte, nämlich man müsse «etwas tun, das gegen die Pflicht verstößt» (DEU 154).

Worin liegt, so können wir uns nun fragen, die Didaktik der Romane? Die stoffliche Fülle, die mitunter humorvollen, ironischen Betrachtungsweisen steigern das Vergnügen am Lesen, doch verdecken sie keineswegs den erhobenen Zeigefinder des Autors. Für Lenz ist Literatur ein «Kommentar zur Welt» und der «Künstler ein Mitwisser von Übelständen unserer Welt», dessen Aufgabe darin besteht, seine Mitwisserschaft zu erkennen zu geben ${ }^{7}$. S. Lenz steht somit in der Nachfolge Lessings, denn seine Intention besteht darin, mit Hilfe seiner sozialkritischen Romane eine kognitive, moraldidaktische Wirkung auf den Leser auszuüben.

Der Roman Deutschstunde soll dem Leser den Mechanismus einer ideologischen Unterwanderung vor Augen führen, die aus einfachen Menschen Komplizen der Zerstörung macht. Er soll zeigen, welche Gefahren eine Idealisierung der sozialen Rolle mit sich bringt und das Mißtrauen gegenüber jeglicher Art von Idealen wecken. In der Gestalt des Polizisten Jepsen wird der Fehler des Idealisten kurzum verdeutlicht, und dieser Fehler heißt Abstraktion. Der Idealist ist unfähig zu erkennen, was das Leben und die Umstände ihn lehren. Er tut, als geschähen die Dinge in der Wirklichkeit so wie sie der Idee nach geschehen müßten, die er sich von der ideellen Welt macht. Er ignoriert also den Abstand, oder den Gegensatz, der die vernünftige, gedachte Welt von der realen Welt trennt. Das aber führt, wie Deutschstunde veranschaulicht, zu einer Pervertierung der höchsten Prinzipien. Menschliches Handeln, und d.h. zeitgeschichtliches Handeln, muß infolgedessen zu kritischem, bewußtem Handeln erzogen werden, so wie es der Autor in Gestalt des Zygmunt in Heimatmuseum darstellt. Dieser zeigt die Notwendigkeit, sich gegebenenfalls von seinen Idealen zu distanzieren. Er illustriert, was der Autor einmal über sich selbst äußert: «Die Lehre hörte nicht auf», schreibt S. Lenz, «ich wollte meine Rolle an einem ganz bestimmten Punkt verstehen: im Augenblick des Widerrufs, der Lossagung, im Moment der Veränderung» ${ }^{8}$. Das 
Distanzieren, das die Romanfigur Zygmunt so heldenhaft vollbringt, wirft jenen aber in einen neuen Konflikt: er verfällt einer chronischen Reflexivität, einem triebhaften Erinnerungszwang über diese für ihn so problematische Aufgabe seiner Rolle, insofern die Vernichtung der individuell gesammelten Museumsschätze nicht nur ihn allein betrifft, sondern gleichzeitig auch ein Eingreifen in kollektives Gut darstellt. Die durch die Gegensatzpaare veranschaulichte Pragmatik der Interaktion zeigt, in welchem Maße die Entscheidung des einzelnen mit der Entscheidung der anderen verbunden ist. Diesbezüglich erinnert Lenz an die Weisheit der Kabbala, «für die Wahrheit das ist, was sich geziemend widerspricht»'. Figur und Gegenfigur bewirken gegenseitiges Kommentieren und rufen zum Kommentar des Lesers auf. Das Lesen der Romane soll somit ein Denken mit fremdem Gehirn auslösen, aber ein Denken in Freiheit, ein Denken, das auf Pluralität gründet und dem Leser keine vorgefaßte Meinung aufzwingen will. In diesem Sinne ist die Didaktik des Autors ein Appell an den Intellekt, an die Reflexion des Lesers. Sie versucht, eine Erkenntnis der jüngsten deutschen Vergangenheit bei ihm hervorzurufen, die auf Vernunft gründet. Sie führt ihm die Mechanismen eines idealisierten Rollenverhaltens und deren verhängnisvolle Folgen objektiv vor Augen und ruft ihn dazu auf, sich affektiv von diesen Rollen zu distanzieren. Denn nur bewußt kritische Distanz kann vor einem mechanischen, auf Gewohnheit gründenden Verhalten und somit vor Entfremdung hüten.

Die Intention des Autors ist eindeutig, die ästhetische Wirkung auf den Leser hingegen weniger, denn sie liegt im Bereich des Möglichen. Es gibt kein Wissen von Reaktionen angesichts literarischer Erfahrungen, so wenig wie es ein Wissen vom Schönen gibt, wie Kant uns lehrt. Dennoch kann man drei mögliche Lesergruppen unterscheiden. Der Leser der ersten Gruppe mag subjektiv vielleicht so weit vom Romangeschehen entfernt sein, daß er entweder erklärt, diese Problematik gehe ihn nichts an, oder aber er erweist sich einfach als unfähig, die sozialkritische Tragweite zu erkennen, was zur Folge hat, daß er sich auf Nebensächlichkeiten begrenzt. Eine zweite Gruppe mag jene Leser betreffen, die sich der realen Gefahren einer Rollenidealisierung für zeitgeschichtliches Denken und Handeln nicht ausreichend bewußt sind, die jedoch fähig sind, die lehrhafte Intention des Autors zu erkennen. In der Tat vermittelt der Autor nicht nur das Wissen von der Notwendigkeit einer Rollendistanzierung; er zeigt vielmehr an Hand des Romangeschehens die Mechanismen auf, die zu diesem Erkennungsakt führen. Vor allem an diese Leser richtet sich der Autor. Die dritte Gruppe mag die aufgeklärten Leser betreffen, jene, die die Gefahren einer Idealisierung erkannt haben und anwenden, was der Autor zu lehren versucht. Jene werden durch die literarische Didaktik in ihren Meinungen bestärkt. Hier aber taucht eine neue Gefahr für das effektive Handeln auf: die Warnungen des Autors können den Leser so sehr in seinem kritischen Bewußtsein bestärken, daß er sich zu sehr distanziert, folglich keine Rolle mehr ernst zu nehmen vermag und vor lauter Bedenken und Kritisieren so verunsichert wird, daß er einer chronischen Reflexivität verfällt, die in sich selbst gefangenbleibt und kein Handeln mehr zu orientieren vermag. Gerade das veranschaulicht Zygmunt in Heimatmuseum, denn das Aufgeben seiner Rolle stürzt ihn in einen triebhaften Erinnerungszwang, dem kein Ende abzusehen ist.

Der literarischen Didaktik sind also notgedrungen Grenzen gesetzt. Entweder bestärkt der Autor den Leser in einem Bewußtsein, das er ohnehin schon erworben hat, nämlich daß man sich von den sozialen Rollen distanzieren müsse, wenn man nicht erneut die gleichen historischen Katastrophen hervorbringen will, oder aber er konfrontiert ihn mit einem neuen Gewissenskonflikt. Somit bleibt die Lehre des Autors eine rückwärts 
gewandte Lehre an den Leser, die zeigt, wie es nicht zu machen ist. Möge der Leser selbst Ausschau halten nach einer neuen, positiven Möglichkeit des Daseins.

Wir können also abschließend folgendes bemerken: wer Literatur prinzipiell als autonom und zugleich als soziales Faktum betrachtet, muß auch die begrenzten Wirkungsmöglichkeiten von Literatur anerkennen. Literatur ist keine Politik. Sie wirkt nicht direkt in das tägliche Leben des einzelnen hinein. Zudem kann sie immer nur die paradigmatischen Erfahrungen eines anderen darstellen und in keinem Fall die praktischen Erfahrungen ersetzen, die ein jeder für sich selbst machen muß. So wie der Geizige von Molière noch nie einen Geizigen von seinem Geiz geheilt hat, so wird auch hier die Didaktik des Autors wohl kaum den ideologiebefangenen Leser von seiner Ideologie zu befreien vermögen. Dennoch besitzt Literatur eine große Macht. Sie besitzt die Macht der Sprache. Sie wirkt auf unsere Vorstellungskraft ein und kann durch eindringliches Wiederholen und Verdichten unsere Bewußtseinsstrukturen mit den Problemen und Konflikten des Tages imprägnieren. Sie kann Klarheit über unsere erlebten Erfahrungen und Offenheit für neue Erfahrungen schaffen. Im vorliegenden Fall kann der Leser nachvollziehen, wie sich die Romanfiguren aus der Spannung zwischen ihren privaten Erlebnissen und dem Gang der Zeitgeschichte erfahren, und er kann die Bewußtseinsprozesse erkennen, die ihre Entscheidungen steuern. Auf diese Bewußtseinsprozesse einzuwirken, darin sieht S. Lenz seine Aufgabe als zeitgenössischer Autor. In diesem Sinne kann Literatur als Denkanstoß zu neuen Möglichkeiten führen und unser zukünftiges Handeln dynamisieren. S. Lenz schreibt: «Gedächtnisarbeit: das heißt auch bekennen, urteilen und für die Gegenwart handeln. Wir müssen dazu bereit sein, denn nur wer handelt, kann hoffen» ${ }^{10}$. Dennoch läßt der Autor in seinen Schriften Skepsis gegenüber den begrenzten Wirkungsmöglichkeiten von Literatur und dem Dichteramt als «pädagogischer Aufgabe» durchblicken.

Zweifel und Hoffnung gehen Hand in Hand. S. Lenz könnte in den Ruf von Erich Kästner einstimmen:

Ich bin der Dichter, der euch anfleht und beschwört, Ihr seid das Volk, das nie auf seine Dichter hört.

\section{NOTES}

1. - Siegfried Lenz: Beziehungen. Ansichten und Bekenntnisse zur Literatur, dtv, 1975, S. 179.

2. - Cf. Alexandre Kojève: Introduction à la lecture de Hegel, Paris, Gallimard, 1947.

3. - Siegfried Lenz: Über das Gedächtnis. Reden und Aufsätze, Hamburg, Hoffmann \& Campe, 1992, S. 40 .

4. - Siegfried Lenz: Deutschstunde, dtv, (Erstveröffentlichung: Hamburg, Hoffmann \& Campe, 1968); in folgenden zitiert: DEU.

5. - Siegfried Lenz: Heimatmuseum, Hamburg, Hoffmann \& Campe, 1978, im folgenden zitiert: HEIM.

6. - Karl Marx/Friedrich Engels: «Kritik des Hegeischen Staatsrechts» in Werke; Bd. 1, Berlin, 1957, S. 249.

7. - Cf. Siegfried Lenz: Beziehungen, S. 204. 


\author{
8. - Ibid., S. 31. \\ 9. - Ibid., S. 49. \\ 10. - S. Lenz: Über das Gedächtnis, S. 19.
}

\title{
RÉSUMÉS
}

Die Romane Deutschstunde und Heimatmuseum von Lenz spielen im Deutschland der Nazizeit und der Nachkriegsjahre. Das pragmatische Anliegen des Autors ist dasjenige, die Motivationen und geistigen Strukturen zu ergründen, die in den Entscheidungsprozessen seiner Romangestalten wirksam sind. Diese beruhen bei ihm auf der Identifizierung mit der sozialen Rolle, die das intersubjektive Handeln orientiert.

Der 1968 veröffentlichte Roman Deutschstunde veranschaulicht die verhängnisvollen Folgen einer Rollenidealisierung an Hand des Konfliktes zwischen dem Polizisten Jepsen und dem expressionistischen Maler Nansen, über den der Nazistaat ein Malverbot verhängt hatte, das der Polizist zu überwachen hat. Jepsens autoritäres Verhalten ist bestimmt durch sein Missionsbewußtsein als Aufklärer des Volkes. Die Pragmatik des Romans läßt die Heranbildung jener Bewußtseinsstrukturen erkennen, die den Faschismus möglich gemacht haben.

Der 1978 veröffentlichte Roman Heimatmuseum zeigt demgegenüber in der Gestalt des Erzählers Zygmunt Rogalla einen ideologiebewußten Menschen, der aus der Geschichte seiner Väter gelernt hat und der Gefahr einer Rollenidealisierung durch krititische Distanz zu entgehen versucht. Im entscheidenden Moment sagt er sich vom Ideal seiner Rolle los und steckt sein Museum in Brand, was ihn jedoch in eine Bewußtseinskrise und in eine chronische Reflexion über die Grenzen von privatem und öffentlichem Handeln wirft.

Die didaktische Intention der Romane besteht darin aufzuzeigen, in welchem. Maße zeitgeschichtliches Handeln durch die Bewußtseinsstrukturen der Handlungsträger bedingt ist und den Leser zu kritischem, bewußtem Handeln zu erziehen. Der ästhetischen Wirkung jedoch sind notwendigerweise Grenzen gesetzt. Als Appell an den Leser bewegt sie sich im Raum des Möglichen, ist aber zugleich immer auch ein Denkanstoß zu neuen Möglichkeiten.

Les romans Deutschstunde (La leçon d'allemand) et Heimatmuseum (Le Musée de la patrie) évoquent l'Allemagne nazie et le temps de l'après-guerre. L'intention pragmatique de l'auteur est d'examiner les motivations et les structures d'esprit opérantes dans les décisions de ses figures romanesques. Celles-ci reposent sur l'identification aux rôles sociaux orientant l'action.

Le roman Deutschstunde, publié en 1968, illustre les conséquences néfastes de l'idéalisation des rôles en narrant le conflit qui oppose le policier Jepsen au peintre expressionniste Nansen. Ce dernier a reçu du régime nazi une interdiction de peindre et le policier Jepsen est chargé de le surveiller. La conduite autoritaire de Jepsen vient de la conscience qu'il a de sa mission et de son désir d'exécuter fidèlement les ordres reçus, celle de Nansen témoigne d'une conscience critique et son désir d'agir comme éclaireur du peuple. La pragmatique du roman met en évidence la complémentarité de ces structures de conscience qui ont rendu possible le pouvoir du fascisme. Le roman Heimatmuseum, publié en 1978, montre au contraire un narrateur, Zygmunt Rogalla, qui a appris de l'histoire de ses pères. Il cherche à garder une distance nécessaire à l'égard de son rôle afin d'échapper au danger de leur idéalisation. Au moment décisif, il ose abandonner l'idéal de son rôle en incendiant le musée qu'il a construit. Ce geste le plonge dans une crise de conscience et une réflexion chronique sur les limites entre l'action privée et de l'action publique. 
L'intention didactique des romans est de montrer à quel point les actions de l'histoire contemporaine dépendent des structures de conscience des actants et à former le lecteur à agir de façon critique et consciente. Les effets esthétiques en sont néanmoins nécessairement limités et ne se meuvent que dans le domaine du possible. Ces romans lancent toutefois un appel au lecteur et l'incitent à réfléchir à d'autres possibilités que celle qu'il a.

\section{AUTEUR}

\section{ELFIE POULAIN}

Université Charles-de-Gaulle - Lille III 